\title{
Research Square \\ Extremely Low Effective Impedance in Stratified Graphene-Dielectric Metamaterials
}

Ruey-Bing Hwang ( $\nabla$ corresponding.raybeam@nycu.edu.tw)

National Yang Ming Chiao Tung University

Research Article

Keywords: reflections, frequency, propagation, interference

Posted Date: October 29th, 2021

DOI: https://doi.org/10.21203/rs.3.rs-1018551/v1

License: (c) (i) This work is licensed under a Creative Commons Attribution 4.0 International License. Read Full License 


\title{
Extremely low effective impedance in stratified graphene-dielectric metamaterials
}

\author{
Ruey-Bing Hwang
}

Institute of Communications Engineering, College of Electrical and Computer Engineering, National Yang Ming

Chiao Tung University, Hsinchu 30050, Taiwan

corresponding.raybeam@nycu.edu.tw

\begin{abstract}
The periodic reflections in frequency were observed in a stack of graphene layers and reported as a series of mini photonic bandgaps owing to the multiple interference by the graphene layers. In this research, the effective medium approach was employed to obtain the effective refractive index and Bloch impedance for understanding the wave propagation characteristic therein. Specifically, the pure real effective refractive index without attenuation as well as an extremely low Bloch impedance were found at the frequencies exhibiting periodic reflections. Some numerical examples were demonstrated to show that the series bandgap-like reflections in fact are attributed to considerable impedance mismatch caused by this ultra low Bloch impedance.
\end{abstract}

\section{Introduction}

Graphene is believed to be one of the most striking materials with its optical property defined only by the fundamental constants rather than on material parameters. The scattering characteristics of a suspended graphene is determined merely by the fine structure constant associated with quantum electrodynamics ${ }^{1}$. Experimental studies indicated that the optical sheet conductance of graphite per graphene layer is very close to the theoretically expected value of dynamical conductance of isolated monolayer graphene ${ }^{2,3}$. Moreover, for few graphene layers $(<6)$, the structure behaves as a superposition of single sheet acting as independent two-dimensional electron gases; the absorptance is proportional to the number of layers ${ }^{4}$. The full expression for the optical conductivity based on the general noninteracting tight-binding model was developed for the scattering analysis of a graphene layer ${ }^{5}$. Having the closed-form expression for graphene optical conductivity, numerical electromagnetic field simulation for the structure consisting of single or multilayered graphene becomes more realistic and sophisticated $^{6,7}$. Due to the reconfiguration of graphene optical conductivity by electrically or magnetically tuning the Fermi level (chemical potential) of a graphene sheet, some potential applications were proposed and implemented; to mention a few, a waveguide-integrated electroabsorption modulator based on monolayer graphene was developed by electrically tuning the Fermi level of the graphene sheet ${ }^{8}$. The Faraday rotation turning the polarization by several degrees through a single- and multilayered graphene was demonstrated in modest magnetic fields ${ }^{9}$. By stacking graphene bearing quartz substrate on a ground plane, an optically transparent broadband absorbers operating in millimeter wave region was achieved ${ }^{10}$. A ultra-broadband absorber made of multilayered graphene metamaterial able to absorb $90 \%$ of the incident wave under normal incidence in the frequency range of 1.12-3.78 $\mathrm{THz}$ was reported ${ }^{11}$. A metamaterial consisting of weakly absorbing alternating graphene layers separated by lossless dielectric was fabricated to serve as a polarization-independent extremely broadband absorber covering almost the entire solar spectrum over a large angular range ${ }^{12}$. A graphene-based tunable hyperbolic metamaterials was designed for enhanced absorption in far-infrared frequencies ${ }^{13}$. Regarding the fabrication technology development, multilayered metamaterial consisting of alternating monolayer graphene oxide/graphene and dielectric layers without a transfer step was successfully developed ${ }^{14}$.

In this research, a 1D metamaterial made of a stratified graphene-dielectric structure is studied. The transfer matrix method was popularly employed to obtain the rigorous solution for scattering analysis ${ }^{15-17}$, while the physical insight has to invoke some other approaches for understanding the wave propagation characteristics. For example, The extraction method for determining the effective index and impedance from the scattering parameters of a finite slab of metamaterial normally incident by a plane wave was developed ${ }^{18}$. The $S$-parameter retrieval was employed to obtain the effective optical properties including permittivity and permeability of the fabricated zero index medium based on purely dielectric constituents ${ }^{19}$. The extraction of the effective medium properties (refractive index and impedance) of symmetric and asymmetric nanoparticle arrays with arbitrary geometry was developed and verified with analytical approach in the limitation of electrical small ${ }^{20}$. The dielectric 


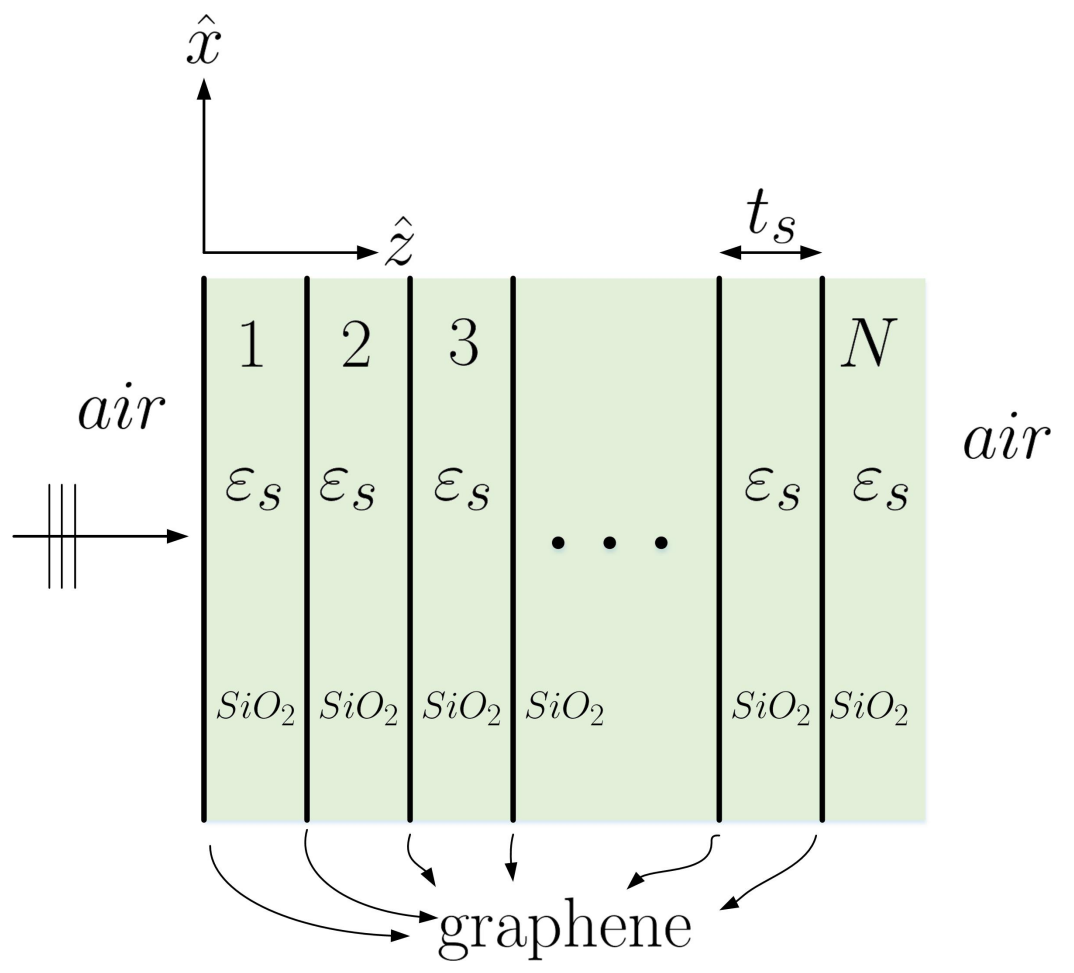

Figure 1. Structure configuration of a stratified graphene-dielectric metamaterial

permittivity tensor of the effective non-local medium with a periodic stack of graphene layers was developed for demonstrating its tunability from elliptic to hyperbolic dispersion with an external gate voltage ${ }^{21}$.

Because an extremely large number of periods (unit cells) are considered here, the dispersion relation of wave propagating in the structure of infinite in extent can be employed to understand the physical insight of wave process. Specifically, the effective medium approach based on Floquet-Bloch theory (periodic boundary condition) is utilized to calculate the effective refraction index and Bloch impedance in the $1 \mathrm{D}$ periodic medium. Consequently, the finite periodic structure can be modeled as a finite length transmission line with effective (average) propagation constant and line impedance. By solving the eigenvalue problem of the transfer matrix of a unit cell, the Bloch impedance can be uniquely determined by the eigenvectors. Moreover, the effective refractive index can be determined by the eigenvalues. Nevertheless, the real part of refractive index runs into multiple-valued problem and has different possible branches, while the imaginary part can be determined unambiguously. The excellent agreement of the scattering analysis between transfer matrix method and effective medium approach allows us to confidently interpret the wave phenomena using the effective refractive index and Bloch impedance.

Specifically, the periodic reflections in frequency occurring in a stratified graphene-dielectric structure were observed ${ }^{15,22}$ and reported as mini stopbands. Through the calculation using effective medium approach, we found that the periodic reflections take place at those frequencies having vanishing imaginary part of effective refractive index and extremely low Bloch impedance. As is well known, the stopband has complex propagation constant in a certain frequency range; the imaginary part is termed as attenuation constant causing decay in incident wave. For a pure real propagation constant with vanishing attenuation constant, it is impossible to support a stopband. In this research, we conclude the strong reflection to be attributed to considerable impedance mismatch between that of source (load) and the extremely low Bloch impedance in effective medium. A limiting case with low source and load impedance was given as an example to demonstrate the significant transmission but low reflection in the same metamaterial.

\section{Structure under consideration}

Figure 1 shows a stratified graphene-dielectric metamaterial. The structure is made of alternating graphene and silica $\left(\mathrm{SiO}_{2}\right)$ having thickness of $t_{s}$ and refractive index designated as $n_{s}$. The graphene sheet is assumed to be zero thickness with the graphene optical conductivity $\sigma_{g}$. The structure is composed of $N$ periods (unit cells) each consisting of a graphene sheet and a $\mathrm{SiO}_{2}$ slab. A plane electromagnetic wave is normally incident into the metamaterial. The graphene and $\mathrm{SiO}_{2}$ slab are assumed to be infinite in extent along the $x-y$ plane. 


\section{Method of mathematical analysis}

\section{Transfer matrix method (TMM)}

The transfer matrix of a graphene sheet with infinite extension along the $x-y$ plane is written as:

$$
\mathbf{T}_{\text {gra }}=\left[\begin{array}{cc}
1 & 0 \\
\sigma_{g} & 1
\end{array}\right],
$$

where $\sigma_{g}$ is the graphene optical conductivity.

Moreover, the transfer matrix of the dielectric slab $\left(\mathrm{SiO}_{2}\right)$ is written as:

$$
\mathbf{T}_{S i O_{2}}=\left[\begin{array}{cc}
\cos k_{z}^{(s)} t_{s} & j Z_{s} \sin k_{z}^{(s)} t_{s} \\
j Y_{s} \sin k_{z}^{(s)} t_{s} & \cos k_{z}^{(s)} t_{s}
\end{array}\right] .
$$

Since the normal incidence is considered, we have $k_{z}^{(s)}=k_{o} n_{s}$ and $Z_{s}=120 \pi / n_{s}$.

The transfer matrix of the unit cell (period) is written as $\mathbf{T}_{\text {cell }}=\mathbf{T}_{\text {gra }} \mathbf{T}_{\mathrm{SiO}_{2}}$. Furthermore, the transfer matrix of a periodic structure consisting of $N$ unit cells can be written as $\mathbf{T}=\left(\mathbf{T}_{\text {cell }}\right)^{N}$. Here, $\mathbf{T}$ is a 2-by-2 matrix. The transmittance (or insertion loss in microwave engineering) denoted as $S_{21}$ can be written as:

$$
S_{21}=\frac{2}{A+B / Z_{o}+C Z_{i}+D Z_{i} / Z_{o}}
$$

Additionally, the reflectance (or termed as return loss) is given as:

$$
S_{11}=\frac{A+B / Z_{o}-C Z_{i}-D Z_{i} / Z_{o}}{A+B / Z_{o}+C Z_{i}+D Z_{i} / Z_{o}}
$$

where $Z_{i}$ and $Z_{o}$ are the input- and output- characteristic impedance in the input and output regions, respectively. Additionally, we have $A=\mathbf{T}(1,1), B=\mathbf{T}(1,2), C=\mathbf{T}(2,1)$, and $D=\mathbf{T}(2,2)$. Notably, parameter $S_{11}$ and $S_{21}$ are defined as the reflectionand transmission-coefficients; they, in general, are complex numbers.

\section{Effective medium approach (EFA)}

For an infinite periodic structure, the wave propagating characteristics can be understood from the property of its unit cell. By the Floquet-Bloch theory, the input-output relation of a unit cell satisfies

$$
\mathbf{T}_{\text {cell }} \mathbf{x}=\chi \mathbf{x}
$$

where $\mathbf{x}$ is a column vector composed of voltage and current amplitudes at the input end, and two eigenvalues $\chi=\exp \left( \pm j \kappa t_{s}\right)$. Parameter $\kappa=\beta-j \alpha$ is the effective propagation constant of the wave propagating through the unit cell. Namely, in such an infinite periodic medium, the wave can propagate in an "average" propagation constant $\kappa$. Therefore, the effective refractive index is defined as $n_{e f f}=\kappa / k_{o}$. Notably, parameter $\kappa$ generally is a complex number due to that of the graphene conductivity $\sigma_{g}$.

Equation (5) is an eigenvalue problem. Having the given parameters in matrix $\mathbf{T}_{c e l l}$, the eigenvalue $\chi$ and eigenvector $\mathbf{x}$ can be readily determined. Furthermore, the Bloch impedance can be written as $Z_{B}=\mathbf{x}(1) / \mathbf{x}(2)$. Notably, two eigenvectors will be obtained, the criterion for choosing the correct $Z_{B}$ is that its real part must be positive. Contrarily, $\chi=\exp \left( \pm j k_{0} n_{e f f} t_{s}\right)$ is a multiple-valued function of $n_{e f f}$. Namely, $k_{0} n_{e f f} t_{s}+q 2 \pi$, where $q \in$ integer (branches) are also their solutions. More specifically, the real- and imaginary-parts of $n_{e f f}=n_{e f f}^{\prime}-j n_{e f f}^{\prime \prime}$ can be determined as follows.

$$
\begin{aligned}
& n_{e f f}^{\prime \prime}= \pm \frac{\ln |\chi|}{2 \pi} \frac{\lambda}{t_{s}} \\
& n_{e f f}^{\prime}= \pm \frac{\phi_{\chi}}{2 \pi} \frac{\lambda}{t_{s}}-q \frac{\lambda}{t_{s}},
\end{aligned}
$$

where eigenvalue $\chi=|\chi| e^{j \phi_{\chi}}$ and $\lambda$ is the operating wavelength.

From Equations (6) and (7), we know that the imaginary part of $n_{e f f}$ can be uniquely determined $\left(n_{e f f}^{\prime \prime} \geq 0\right.$ for passive medium), while the real part of $n_{\text {eff }}$ accommodates multiple values. 


\section{Graphene optical conductivity}

Graphene conductivity $\left(\sigma_{g}=\sigma_{\text {intra }}+\sigma_{\text {inter }}\right)$, having a close-form expression for the condition $\left|\mu_{c}\right| \gg k_{B} T$, consists of both the intraband $\left(\sigma_{\text {intra }}\right)$ and inter-band $\left(\sigma_{\text {inter }}\right)$ terms: ${ }^{6}$

$$
\begin{aligned}
& \sigma_{\text {intra }}(\omega)=\frac{-2 j e^{2} k_{B} T}{\pi \hbar^{2}(\omega-j \gamma)} \ln \left[2 \cosh \left(\mu_{c} / 2 k_{B} T\right)\right], \text { and } \\
& \sigma_{\text {inter }}=\frac{e^{2}}{4 \hbar}\left\{\frac{1}{2}+\frac{1}{\pi} \arctan \frac{\hbar(\omega-j \gamma)-2 \mu_{c}}{2 k_{B} T}+\frac{j}{2 \pi} \ln \frac{\left[\hbar(\omega-j \gamma)+2 \mu_{c}\right]^{2}}{\left[\hbar(\omega-j \gamma)-2 \mu_{c}\right]^{2}+\left(2 k_{B} T\right)^{2}}\right\},
\end{aligned}
$$

where $-e$ is the electron charge, $\hbar$ is the reduced Planck constant, $\gamma$ is a phenomenological carrier scattering rate $\left(\gamma=1 / 2 \tau_{c}\right.$, where $\tau_{c}$ is the carrier relaxation time), $\mu_{c}$ is the chemical potential, $k_{B}$ is Boltzmann's constant, and $T$ is the ambient temperature.

\section{Numerical Results and Discussion}

Before the elaborate calculations, we have to first understand the graphene optical conductivity against frequency. Here, the normalized angular frequency $\Omega$ is defined as: $\Omega=\omega \hbar / 2 \mu_{c}^{(o)}$, where we have $\mu_{c}^{(o)}=0.35 \mathrm{eV}$ throughout this paper. In the vicinity of $\Omega=1$, the change in $\sigma_{g}$ is obvious for the variation in $\mu_{c}$. The operating frequency is selected in this region to see the influence of conductivity variation on scattering characteristics of the graphene metamaterial under consideration. Figures 2(a) and 2(b) respectively show the real- and imaginary-part of graphene optical conductivity due to the summation of Equations 8 and 9 against $\Omega$ for various chemical potential while having a fixed relaxation time $\tau_{c}=0.03 \mathrm{ps}$.

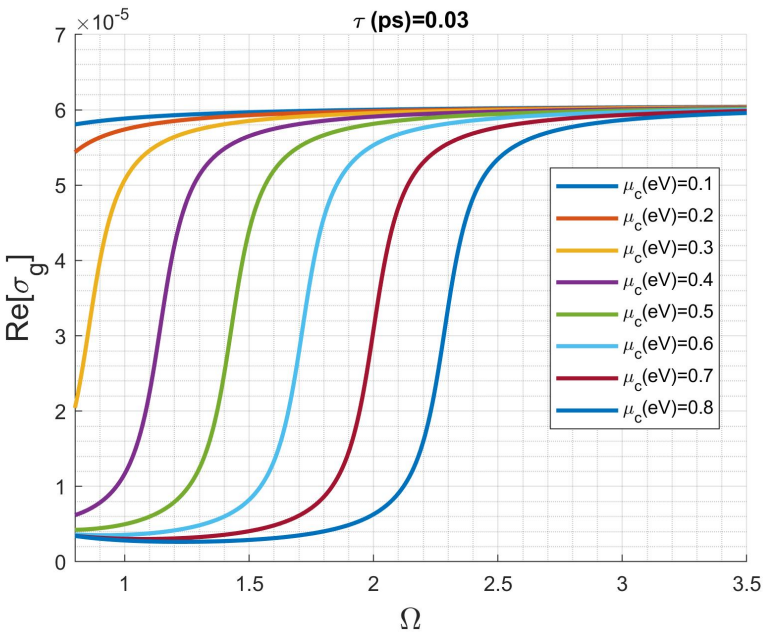

(a)

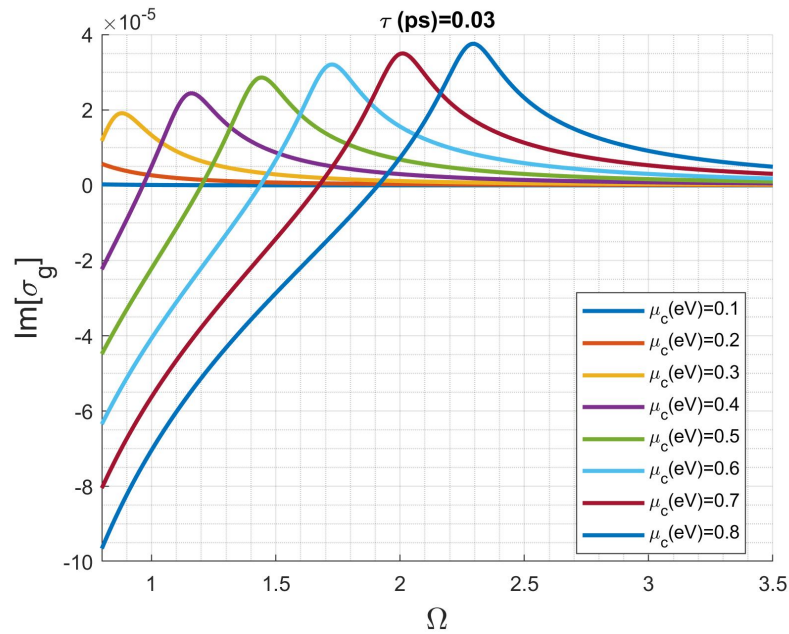

(b)

Figure 2. Graphene optical conductivity versus normalized angular frequency $\Omega=\hbar \omega / 2 \mu_{c}^{(o)}$ (where $\mu_{c}^{(o)}=0.35 \mathrm{eV}$ ) for various chemical potential $\mu_{c}$ : (a) real part and (b) imaginary part. The carrier relaxation time $\tau_{c}=0.03 \mathrm{ps}$.

Figures 3(a) and 3(b) are the scattering characteristics including transmittance, reflectance and absorptance against normalized angular frequency through the rigorous calculation by TMM. It is interesting to observe the frequency-selective reflection and transmission corresponding to the spikes shown in Figure 3(a). In addition to the strong reflection, the periodic absorption dips are also found at $\Omega=1.33333 \mathrm{~m}$, where $m$ is an integer starting from unity. Figure 3(b) shows the detailed view of frequency-selective behavior at the first dip around $\Omega=1.33333$. The strong reflection is conventionally explained as due to stop-band, causing the significant reduction in absorption by the graphene sheets. As far as a stopband is concerned, the dispersion relation or band structure must be carefully examined to give a solid proof.

In Figure 4, the magnitude of transmittance $\left(S_{21}\right)$ and reflectance $\left(S_{11}\right)$ versus normalized angular frequency for both aforementioned approaches were evaluated. The exact results presented in curves are obtained by TMM, while those in symbols are obtained by the EMA. Notably, the first peak is at $\Omega=1.33333$ corresponding operating wavelength of $\lambda=1771.203 \mathrm{~nm}$; 


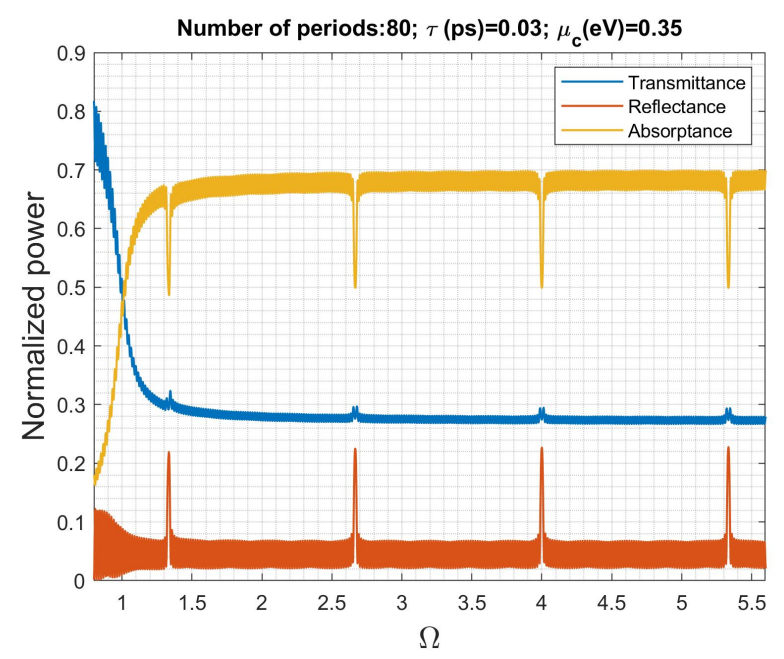

(a)

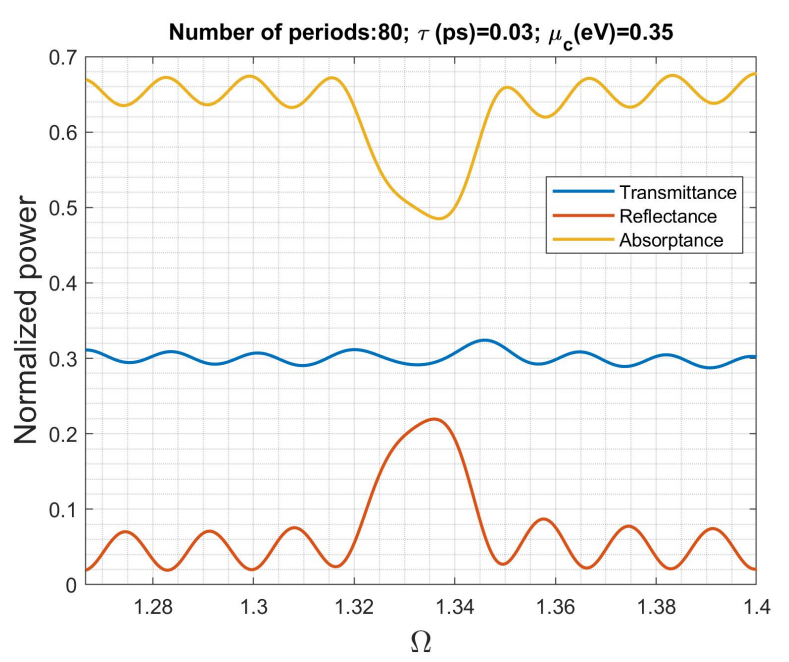

(b)

Figure 3. Scattering efficiency (normalized power) including transmittance, reflectance and absorptance versus normalized angular frequency $(\Omega)$. The number of unit cells (periods) is 500. The graphene sheet has the following parameters: $\mu_{c}=0.35 \mathrm{eV}$ and $\tau=0.03 \mathrm{ps}$. The dielectric slab is $\mathrm{SiO}_{2}$ having refractive index $n_{s}=1.5$ and thickness of $442.8007 \mathrm{~nm}$. (a) global view and (b) in the vicinity of the first normalized frequency with absorption dip at $\Omega=1.33333$.

the thickness of unit cell (period) is $t_{s}=442.801 \mathrm{~nm}$. Consequently, it can be regarded as a continuous medium around this frequency. Due to the multiple-valued function of $\Re\left[n_{e f f}\right]$ in Equation 7, three branches of $q=0, q=-1$ and $q=+1$ are considered to calculate the scattering property of the effective medium. Although only the three branches are shown here, all the integers $q$ in Equation 7 can achieve the same result as the TMM does, even for the operating frequency beyond the assumption of continuous medium.

\section{Extremely low effective Bloch impedance}

Figures 5(a) and 5(b) show the effective refractive index $\left(n_{e f f}\right)$ and Bloch impedance $\left(Z_{B}\right)$ of the infinite periodic medium with the same unit cell parameters given in the previous examples. Specifically, their behavior in the vicinity of $\Omega=1.33333$ is carefully inspected. In Figure 5(a), the real part of $n_{\text {eff }}$ can be different values in accordance with the integer $q$ defined in Equation (7) due to multiple-valued function of eigenvalue. Significantly, its imaginary part is almost zero $\left(-1.36844 \times 10^{-10}\right)$ at $\Omega=1.33333$. Additionally, one also examines the variation of Bloch impedance around this point. In Figure 5(b), both the real- and imaginary-part of $Z_{B}$ are very small at $\Omega=1.33333$; its reactance is changing from capacitive into inductive through this turning point. The Bloch impedance is uniquely determined independent of the integer $q$. Although only the detailed view of Bloch impedance around $\Omega=1.33333$ is depicted, in fact they share almost the same behavior for the other dips at $\Omega=1.33333 m$, where $m$ is an integer starting from unity.

To explain the zero imaginary part of $n_{e f f}$, we return to the eigenvalue problem in Equation 5. Its alternative expression (the characteristic equation of matrix $\mathbf{T}_{\text {cell }}$ ) can be written below.

$$
\cos \left(k_{o} n_{e f f} t_{s}\right)=\cos \left(k_{o} t_{s} n_{s}\right)+j \frac{\sigma_{g} Z_{o}}{2 n_{s}} \sin \left(k_{o} t_{s} n_{s}\right)
$$

Equation (10) is also termed as the dispersion relation of wave propagating in the 1D periodic medium. The effective refractive index $\left(n_{e f f}\right)$ can be resolved once the parameters including $k_{o}, n_{s}$ and graphene conductivity $\left(\sigma_{g}\right)$ are given. We first consider the condition of $k_{o} t_{s} n_{s}=m \pi$, where $m$ is an integer excluding zero, enabling $\sin \left(k_{o} t_{s} n_{s}\right)=0$. Consequently, we have $\cos \left(k_{o} n_{e f f} t_{s}\right)= \pm 1 ; n_{e f f}$ is a pure real number. It means that the wave propagating in the medium at this frequency has no attenuation.

Additionally, the zero $\mathfrak{I}\left[n_{e f f}\right]$ at $k_{o} t_{s} n_{s}=m \pi$ corresponds to the angular frequency $\omega_{m}=C m \pi / n_{s} t_{s}$, where $C$ is the speed of light. The normalized angular frequency is $\Omega_{m}=m \hbar C \pi / 2 n_{s} t_{s} \mu_{c}^{(o)}$. Substitution of $\mu_{c}^{(o)}=0.35 \mathrm{eV}, n_{s}=1.5$ and $t_{s}=442.8007 \mathrm{~nm}$ into $\Omega_{m}$, we obtain $\Omega_{m}=1.33333 \mathrm{~m}$.

Although $\Re\left[n_{e f f}\right]$ can accommodate multiple values, the eigenvector to Equation 5 is absolutely not. Notably, the 


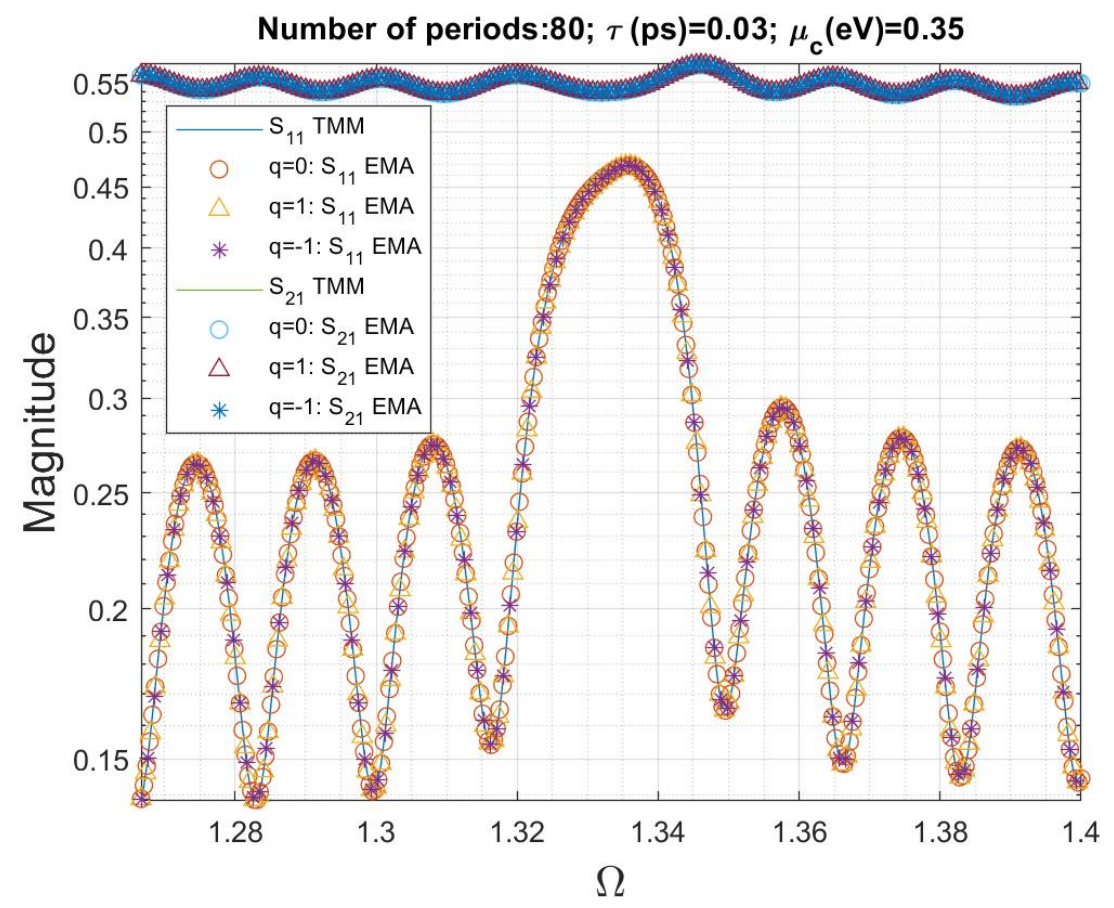

Figure 4. Comparison of the transmittance $\left(S_{21}\right)$ and reflectance $\left(S_{11}\right)$ between transfer matrix method (TMM) and Effective medium approach (EFA).

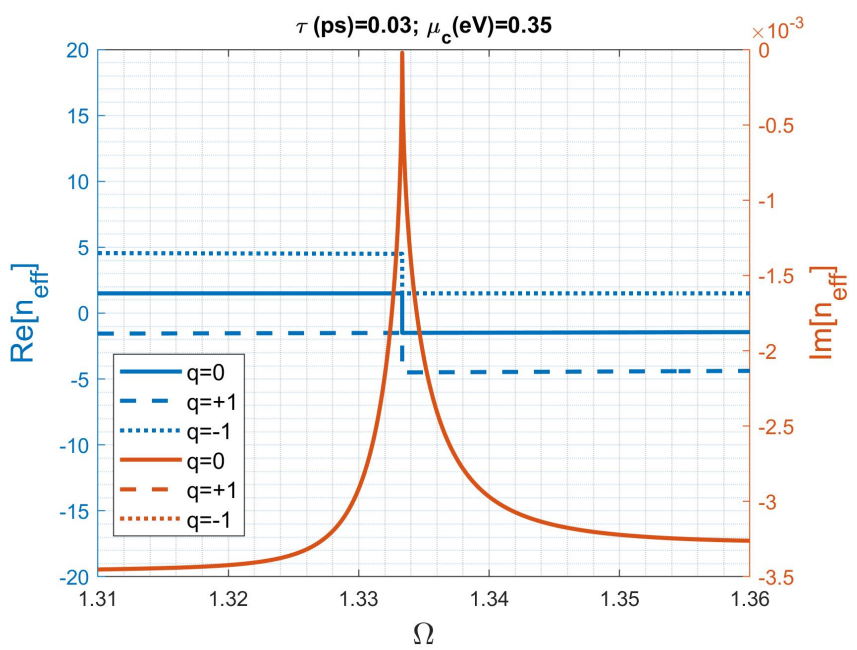

(a)

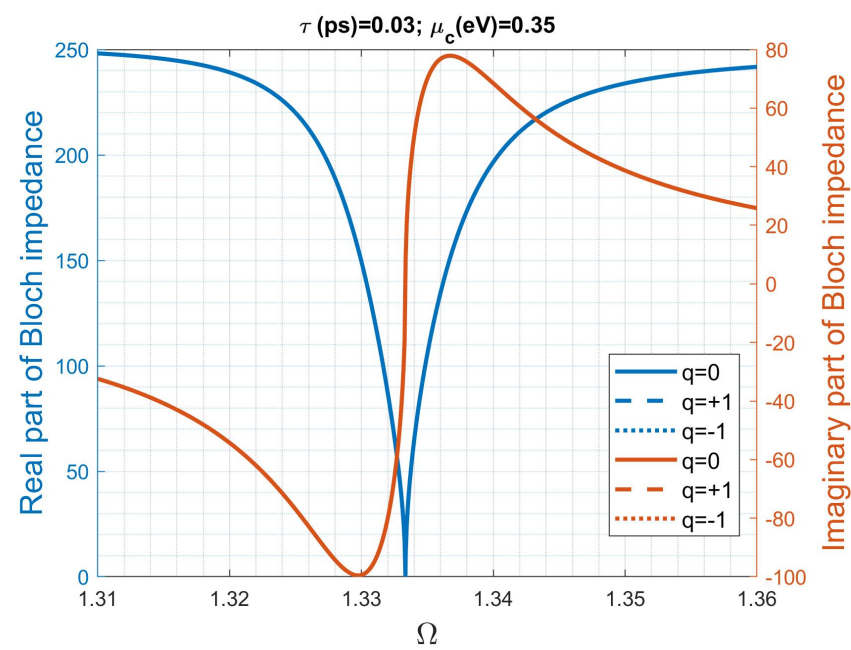

(b)

Figure 5. Effective refractive index and Bloch impedance against normalized angular frequency at around the first minimum absorption frequency $\Omega=1.3333$ : (a) the real- and imaginary-parts of the effective refractive index $\left(n_{e f f}\right)$, and (b) the real- and imaginary-parts of the Bloch impedance $\left(Z_{B}\right)$. 
eigenvectors at the condition of vanishing $\mathfrak{I}\left[n_{\text {eff }}\right]$ are repeated eigenvalues problem and should be carefully evaluated. Specifically, the Bloch impedance is a very small complex number.

The effective refractive index $n_{\text {eff }}$ has negligible imaginary part at the spikes, the complex Bloch impedance enables the power absorption by the effective medium due to the presence of graphene sheets.

Figure 6(a) and 6(b) individually depict the effective refractive index and Bloch impedance versus normalized angular frequency with the same range shown in Figure 2(a). It is obvious to see that the frequencies with transmission/reflection spikes coincide with those having negligible $\operatorname{Im}\left[n_{e f f}\right]$. Additionally, those peaks with vanishing $\mathfrak{I}\left[n_{e f f}\right]$ occur at $\Omega=1.33333 m$, where $m$ is ranging from 1 to 4 . Because of multiple-valued function of $\Re\left[n_{e f f}\right]$, all the integer $q$ should be taken into account. However, in this figure only the three branches: the blue solid, dashed and dotted curves individually corresponding to branches of $q=0, q=+1$ and $q=-1$, are plotted. On the other hand, their imaginary part share the same distribution. In the low frequency region with $\Omega<<1\left(t_{s}<<\lambda\right)$, the branches of $q \neq 0$ are away from the principal branch of $q=0$; there is no ambiguity in selection of the correct branch $(q)$. However, it crates difficulty in unambiguously determining the correct branch when $\Omega>1$ since the $\Re\left[n_{e f f}\right]$ of the three branches gradually lie quit close.

Additionally, the Bloch impedance versus normalized angular frequency is also plotted shown in Figure 6(b). There is no ambiguity on the Bloch impedance; thus all the cases of different $q$ coincide with one another. Significantly, the Bloch impedance at $\Omega=1.33333, \Omega=2.66666, \Omega=3.99999$, and $\Omega=5.33333$ are $Z_{B}=4.381 \times 10^{-5}-j 5.052 \times 10^{-5}$, $Z_{B}=3.692 \times 10^{-5}+j 3.650 \times 10^{-5}, Z_{B}=6.669 \times 10^{-5}-j 6.691 \times 10^{-5}$, and $Z_{B}=9.155 \times 10^{-5}-j 9.169 \times 10^{-5}$, respectively.

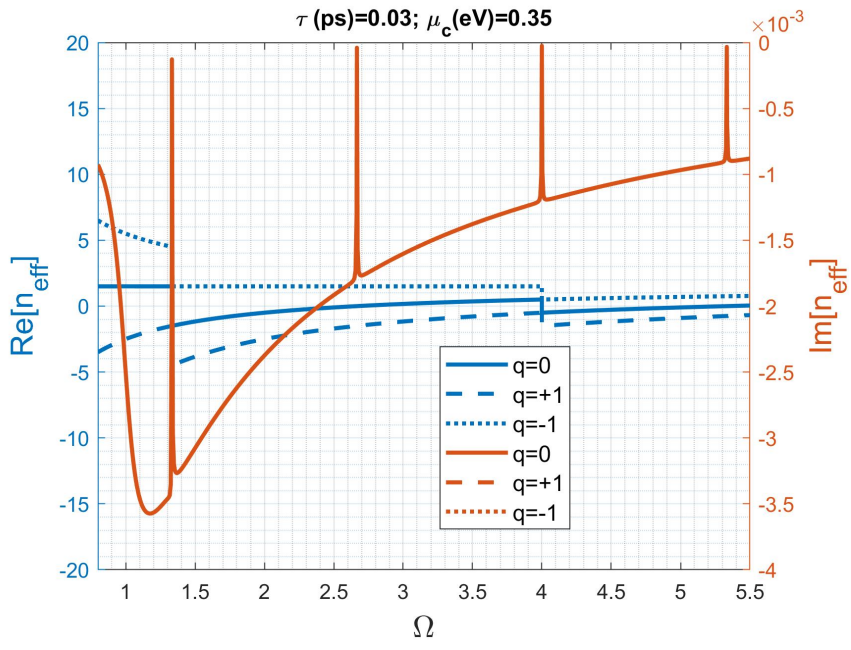

(a)

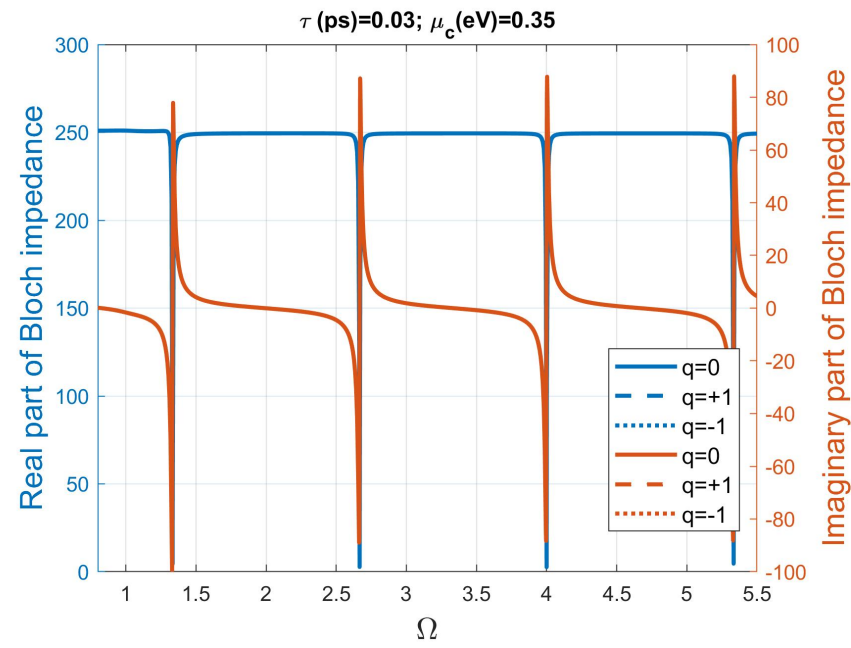

(b)

Figure 6. Effective refractive index and Bloch impedance against normalized angular frequency of the global view: (a) the real- and imaginary-parts of the effective refractive index $\left(n_{e f f}\right)$, and (b) the real- and imaginary-parts of the Bloch impedance $\left(Z_{B}\right)$.

As is well known in a 1D periodic structure, the stop-band is due to the coherent reflection from each unit cell. If we evaluate the dispersion relation of the $1 \mathrm{D}$ wave propagating in an infinite periodic medium, the propagation constant is a complex number $(\kappa=\beta-j \alpha)$ in the stop-band regions, while it is a real number in the pass-band regions. Returning to Figure 6(a), we found that $n_{e f f}$ is a real number at $\Omega=1.33333$. It means no attenuation (or $\alpha=0$ ) present in the 1D wave, unlike the conventional stop-band with significant $\alpha$. Specifically, the Bloch impedance at this frequency is a near-zero complex number; it accounts for the periodic reflection as well as absorption of incident power by the complex graphene conductivity.

Furthermore, the effect of graphene optical conductivity due to chemical potential $\left(\mu_{c}\right)$ change on the absorptance is also studied and the results are shown in Figures 7(a) and 7(b). The power absorption by a graphene sheet is determined by $P_{a b s}=0.5 \Re\left[\mathbf{E} \cdot \sigma_{g r a}^{*} \mathbf{E}^{*}\right]=\sigma_{g r a}^{\prime}|\mathbf{E}|^{2} / 2$, where $\mathbf{E}$ is the electric field on the graphene sheet surface and $\sigma_{g r a}^{\prime}$ is the real part of graphene optical conductivity. Consequently, when compared Figure 7(a) with Figure 2(a), the absorptance versus normalized angular frequency can reveal the profile of $\sigma_{g}^{\prime}$. Because the frequencies of $\Omega_{m}=1.33333 m(m=1,2,3$, and 4$)$ correspond to absorption dips as described previously, the drastic change in the absorptance can be seen. The region around $\Omega=1.33333$ was enlarged to carefully observe their change in each $\mu_{c}$. As depicted in Figure 7(b), the cases of $\mu_{c}=0.7$ and $\mu_{c}=0.8$ have obvious drop in absorptance in accordance with the decrease in $\sigma_{g r a}^{\prime}$. Moreover, the absorptance for the cases with $\mu_{c}$ changing from $0.1 \mathrm{eV}$ to $0.3 \mathrm{eV}$ is not obvious since their variation in $\sigma_{g r a}^{\prime}$ is little. Contrarily, in the second dip at $\Omega=2.66666$, shown in 


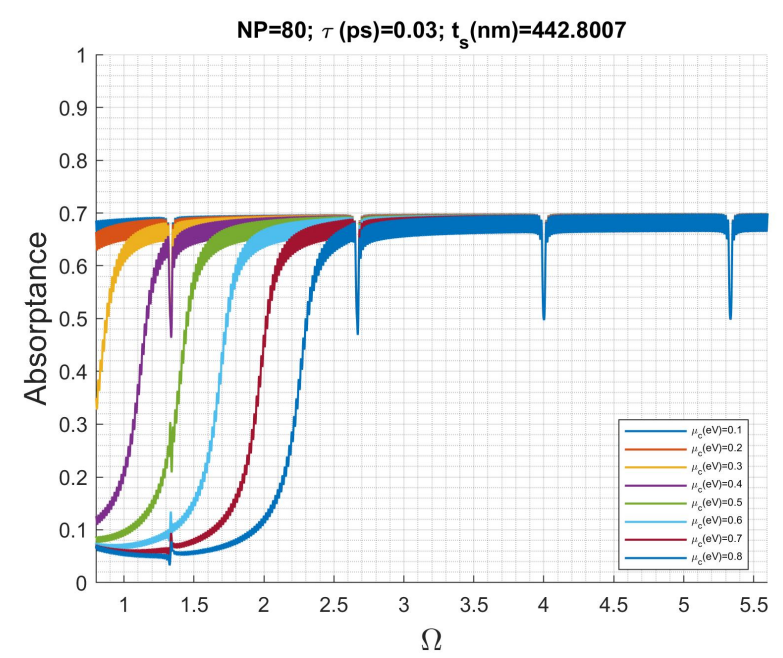

(a)

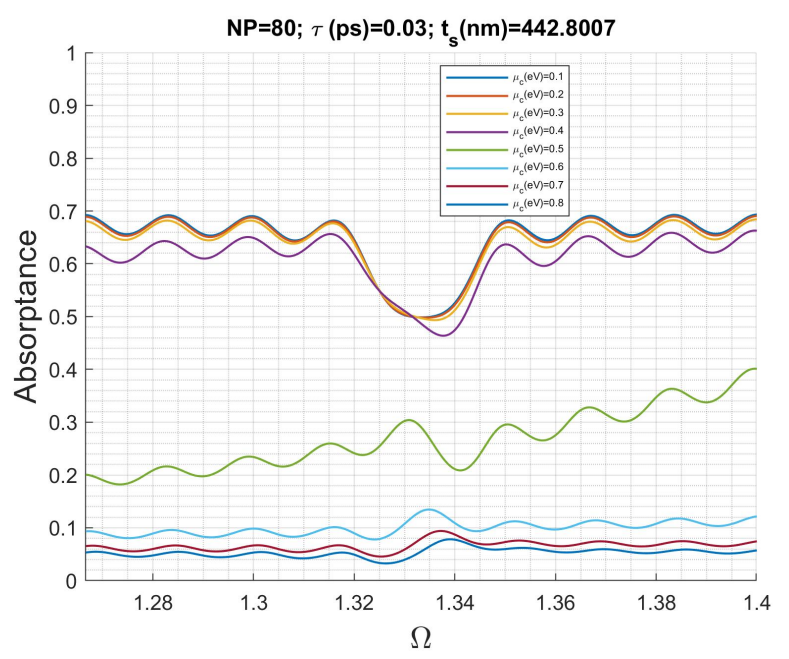

(b)

Figure 7. Absorptance against the normalized angular frequency $(\Omega)$ for various chemical potential (a) global view, and (b) in the vicinity of the first absorption dip.

Fif. 7(a), almost all the cases coincide to one another. It is because of the insignificant change in the $\sigma_{g r a}^{\prime}$ in Figure 2 (a) for all $\mu_{c}$.

In Figure 8, we change the chemical potential to see its influence on the Bloch impedance. The first three normalized frequencies: $\Omega_{1}=1.33333, \Omega_{2}=2.66666$, and $\Omega_{3}=3.99999$ are considered. Return to Figure 2(a), in the vicinity of $\Omega_{1}=1.33333 \Re\left[\sigma_{g}\right]$ has insignificant change for $\mu_{c}$ ranging from $0.1 \mathrm{eV}$ to $0.3 \mathrm{eV}$. Big changes occur at the three cases including $0.4 \mathrm{eV}, 0.5 \mathrm{eV}$, and $0.6 \mathrm{eV}$. The difference between the cases of $0.7 \mathrm{eV}$ and $0.8 \mathrm{eV}$ is inconsiderable. The aforementioned trend in the change of $\Re\left[\sigma_{g}\right]$ also reflects the change in real- and imaginary-parts of $Z_{B}$, shown in Figure 8. Contrarily, for the cases of $\Omega_{2}=2.66666$ and $\Omega_{2}=3.99999$, their variations on $\sigma_{g}$ due to various $\mu_{c}$ are not obvious shown in Figures 2(a) and 2(b). This is the reason why their Bloch impedance have insignificant change depicted in Figure 8. Alternatively, the Bloch impedance (at the condition of $k_{o} n_{s} t_{s}=m \pi$ ) is determined by the eigenvector of the matrix $T_{g r a}$ in Equation 1, which merely depends on graphene optical conductivity $\sigma_{g}$.

To demonstrate that impedance mismatch affects the scattering characteristic, we further reduce the wave impedance in the input and output regions from $377 \Omega$ to $11.922 \Omega$ and carry out the scattering analysis. Notably, the structure parameters of the graphene metamaterial remains the same. Apparently, the transmittance increases while the reflectance decreases dramatically, shown in Figure 9. If it indeed is operated in the stop-band region, the transmittance can not be improved anyway. This simulation allows us to know the impedance match between the Bloch- and source (load)-impedance has a significant influence on the scattering properties.

\section{Conclusion}

The effective medium approach was developed based on the Floquet-Bloch theory to obtain the effective refractive index and Bloch impedance of the metamaterial under consideration. Moreover, a comprehensive comparison in scattering analysis between that of transfer matrix method and effective medium approach is carefully verified. Although multiple branches were obtained in the real part of effective refractive index due to multiple-valued problem, there is no ambiguity in determining the imaginary part of effective refractive index and the Bloch impedance in the effective medium approach. It is interesting to find the periodic stopband-like reflections occur at the conditions of pure real effective refractive index and ultra low Bloch impedance. Moreover, the periodic frequencies depend on the periodicity and refractive index of the dielectric medium rather than graphene. We may conclude that those periodic reflections were caused by extremely impedance mismatch between that of source (load) and effective medium.

\section{References}

1. Nair, R. R. et al. Fine structure constant defines visual transparency of graphene. Science 320, 1308-1308, DOI: 10.1126/science.1156965 (2008). 


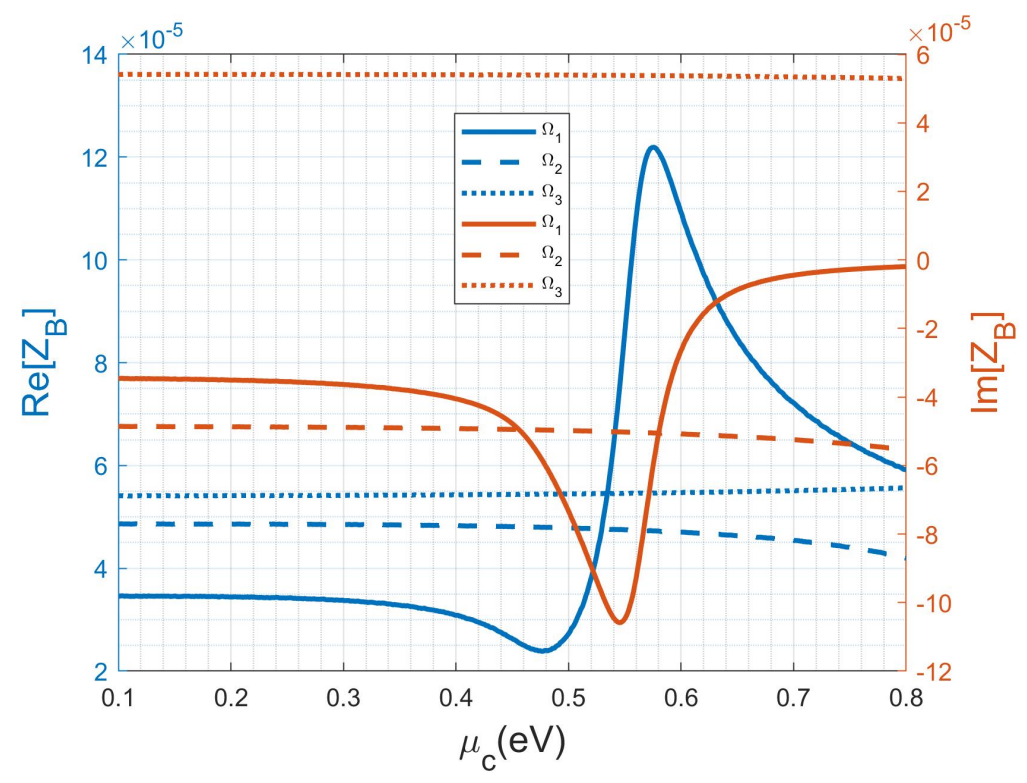

Figure 8. Variation of the Bloch impedance $\left(Z_{B}\right)$ against chemical potential $\left(\mu_{c}\right)$ for the first three modes with normalized frequencies $\Omega_{1}=1.33333, \Omega_{2}=2.66666$, and $\Omega_{3}=3.99999$, respectively.

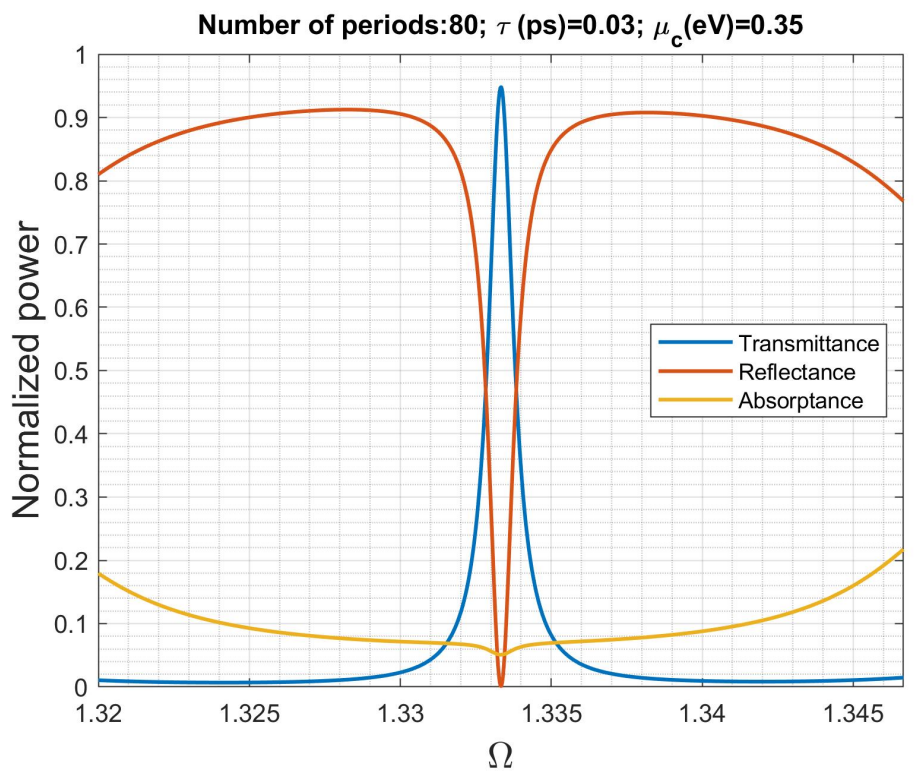

Figure 9. The scattering characteristics of the same $1 \mathrm{D}$ metamaterial but with the wave impedance equal to $11.922 \Omega$ in the input and output region; the original one is $377 \Omega$. 
2. Gusynin, V. P., Sharapov, S. G. \& Carbotte, J. P. Unusual microwave response of dirac quasiparticles in graphene. Phys. Rev. Lett. 96, 256802, DOI: 10.1103/PhysRevLett.96.256802 (2006).

3. Kuzmenko, A. B., van Heumen, E., Carbone, F. \& van der Marel, D. Universal optical conductance of graphite. Phys. Rev. Lett. 100, 117401, DOI: 10.1103/PhysRevLett.100.117401 (2008).

4. Casiraghi, C. et al. Rayleigh imaging of graphene and graphene layers. Nano Lett. 7, 2711-2717, DOI: 10.1021/nl071168m (2007). PMID: 17713959.

5. Stauber, T., Peres, N. M. R. \& Geim, A. K. Optical conductivity of graphene in the visible region of the spectrum. Phys. Rev. B 78, 085432, DOI: 10.1103/PhysRevB.78.085432 (2008).

6. Depine, R. A. Electromagnetics of graphene. In Graphene Optics: Electromagnetic Solution of Canonical Problems, 2053-2571, 1-1 to 1-16, DOI: 10.1088/978-1-6817-4309-7ch1 (Morgan and Claypool Publishers, 2016).

7. Depine, R. A. Graphene gratings. In Graphene Optics: Electromagnetic Solution of Canonical Problems, 2053-2571, 4-1 to 4-23, DOI: 10.1088/978-1-6817-4309-7ch1 (Morgan and Claypool Publishers, 2016).

8. Liu, M. et al. A graphene-based broadband optical modulator. Nature 474, 64-67, DOI: 10.1038/nature10067 (2011).

9. Crassee, I. et al. Giant faraday rotation in single- and multilayer graphene. Nat. Phys. 7, 48-51, DOI: 10.1038/nphys1816 (2011).

10. Wu, B. et al. Experimental demonstration of a transparent graphene millimetre wave absorber with 28 percent fractional bandwidth at $140 \mathrm{ghz}$. Sci. Reports 4, 4130 (2014).

11. Liu, L., Liu, W. \& Song, Z. Ultra-broadband terahertz absorber based on a multilayer graphene metamaterial. J. Appl. Phys. 128, 093104, DOI: 10.1063/5.0019902 (2020).

12. Lin, H. et al. A 90-nm-thick graphene metamaterial for strong and extremely broadband absorption of unpolarized light. Nat. Photonics 13, 270-276, DOI: 10.1038/s41566-019-0389-3 (2019).

13. Othman, M. A. K., Guclu, C. \& Capolino, F. Graphene-based tunable hyperbolic metamaterials and enhanced near-field absorption. Opt. Express 21, 7614-7632, DOI: 10.1364/OE.21.007614 (2013).

14. Yang, Y. et al. Graphene-based multilayered metamaterials with phototunable architecture for on-chip photonic devices. ACS Photonics 6, 1033-1040, DOI: 10.1021/acsphotonics.9b00060 (2019).

15. Zhan, T., Shi, X., Dai, Y., Liu, X. \& Zi, J. Transfer matrix method for optics in graphene layers. IOP Publ. 25, 215301, DOI: 10.1088/0953-8984/25/21/215301 (2013).

16. Hwang, R.-B. R. Periodic Structures: Mode-Matching Approach and Applications in Electromagnetic Engineering (Wiley-IEEE, The address, 2012), 1 edn. In Chapter 6, section 6.1: Scattering characteristics of plane wave by a 1D periodic structure consisting of a cavities array, Chapter 6.

17. Zhang, Y., Wu, Z., Cao, Y. \& Zhang, H. Optical properties of one-dimensional Fibonacci quasi-periodic graphene photonic crystal. Opt. Commun. 338, 168-173, DOI: 10.1016/j.optcom.2014.10.059 (2015).

18. Smith, D. R., Schultz, S., Markoš, P. \& Soukoulis, C. M. Determination of effective permittivity and permeability of metamaterials from reflection and transmission coefficients. Phys. Rev. B 65, 195104, DOI: 10.1103/PhysRevB.65.195104 (2002).

19. Moitra, P. et al. Realization of an all-dielectric zero-index optical metamaterial. Nat. Photonics 7, 791-795, DOI: 10.1038/nphoton.2013.214 (2013).

20. Dyck, N. C., Denomme, R. C. \& Nieva, P. M. Effective medium properties of arbitrary nanoparticle shapes in a localized surface plasmon resonance sensing layer. The J. Phys. Chem. C 115, 15225-15233, DOI: 10.1021/jp203150n (2011).

21. Iorsh, I. V., Mukhin, I. S., Shadrivov, I. V., Belov, P. A. \& Kivshar, Y. S. Hyperbolic metamaterials based on multilayer graphene structures. Phys. Rev. B 87, 075416, DOI: 10.1103/PhysRevB.87.075416 (2013).

22. Bludov, Y. V., Peres, N. M. R. \& Vasilevskiy, M. I. Unusual reflection of electromagnetic radiation from a stack of graphene layers at oblique incidence. IOP Publ. 15, 114004, DOI: 10.1088/2040-8978/15/11/114004 (2013).

\section{Acknowledgements}

This work was supported by the Ministry of Science and Technology (MOST) of Taiwan under grant MOST 110-2221-E-A49020 . 


\section{Author contributions statement}

R.B. completes all the research works and wrote the manuscript.

\section{Additional information}

Competing financial interests: The author declares no competing financial interests. 